\title{
TUBERCULOSE: ANÁLISE DE VÍDEOS DO YOUTUBE
}

\author{
TUBERCULOSIS: ANALYSIS OF THE YOUTUBE VIDEOS
}

\author{
Júlia Andrade Farias ${ }^{1}$ \\ Ana Paula Rocha Sá ${ }^{2}$ \\ Gabrielle Letícia Albuquerque ${ }^{3}$ \\ Mariana Pinheiro de Sousa ${ }^{4}$ \\ Nicolly Andrade Cavalcanti ${ }^{5}$ \\ Mayara Lima Barbosa ${ }^{6}$
}

RESUMO: OBJETIVO: Analisar vídeos no sítio de compartilhamento YouTube, observando os pontos tratados relacionados à tuberculose com base no Manual de recomendações para o controle da tuberculose no Brasil. MÉTODO: Trata-se de uma pesquisa exploratória, com abordagem quantitativa, realizada no sítio YouTube a partir da análise de vídeos sobre a tuberculose, relacionando ao Manual de recomendações para o controle da tuberculose no Brasil. RESULTADOS: A amostra final foi composta por 157 videos. A maioria do material incluído apresentou caráter jornalístico, de autoria de empresas, sobretudo de jornais, apresentando o tratamento e a prevenção dessa patologia como os temas mais prevalentes. CONCLUSÃO: A plataforma digital constitui-se uma importante ferramenta para a disseminação de informações, contudo, é essencial que haja melhor avaliação quanto às informações que são divulgadas, a fim de potencializar a disseminação de conteúdos verídicos e claros.

Palavras chave: Educação para a saúde. Mídia audiovisual. Tuberculose.

ABSTRACT: OBJECTIVE: To analyze videos on the Youtube platform, carefully observing the points addressed in the the topic of Tuberculosis, based on the Manual of Recommendations for Tuberculosis Control in Brazil. METHOD: The present study is an exploratory research, which includes both qualitative and quantitative nature, carried out on the Youtube website based on the analysis of videos about

\footnotetext{
${ }^{1}$ Acadêmico do curso de Medicina/Centro Universitário Unifacisa de Campina Grande.

${ }^{2}$ Acadêmico do curso de Medicina/Centro Universitário Unifacisa de Campina Grande.

${ }^{3}$ Acadêmico do curso de Medicina/Centro Universitário Unifacisa de Campina Grande.

${ }^{4}$ Acadêmico do curso de Medicina/Centro Universitário Unifacisa de Campina Grande.

${ }^{5}$ Acadêmico do curso de Medicina/Centro Universitário Unifacisa de Campina Grande.

${ }^{6}$ Docente do curso de Medicina/Centro Universitário Unifacisa de Campina Grande.
} 
Tuberculosis, relating their aspects to the Manual of Recommendations for Tuberculosis Control in Brazil. RESULTS: The final sample consisted of 157 videos. The majority of the videos had a journalistic nature, authored by companies, especially newspapers ones, presenting the treatment and prevention of this pathology as the most prevalent themes. CONCLUSION: The Youtube platform is an important tool for the dissemination of information, however it is essential that a better assessment of the information that is disclosed is made, in order to enhance the dissemination of truthful and clear content.

Keywords: Health Education. Video-audio Media. Tuberculosis. 


\section{INTRODUÇÃO}

A tuberculose (TB) é uma doença causada por qualquer espécie do gênero Mycobacterium, sendo a mais importante o M. tuberculosis ou Bacilo de Koch. Sendo esta doença uma das principais causas de morte por infecção em adultos em todo o mundo (WHO, 2019).

Conhecida no século XIX, como peste branca, a TB dizimou centenas de milhares de pessoas em todo o mundo. A capacidade do organismo de estabelecer com eficiência a infecção latente permitiu a sua disseminação para quase um terço dos indivíduos em todo o mundo, mas ao longo dos séculos subsequentes houve redução da incidência e da mortalidade relacionadas à $T B$, principalmente pela melhoria das condições de vida das populações (SAAVACOOL, 1986).

Entretanto, por volta da década de 1980, acentuou-se nos países ricos, devido à emergência da infecção pelo Vírus da Imunodeficiência Humana (HIV) e, nos países de baixa renda, devido à horizontalização da miséria, urbanização descontrolada e desestruturação dos serviços de saúde (BLOOM, 1992; CDC, 1993; ROSSMAN; MACGREGOR, 1995).

Globalmente, foi estimado que 10,0 milhões (variação: 9,0-11,1 milhões) de pessoas adoeceram de TB em 2018, um número relativamente estável nos últimos anos, com alta variação entre os países, em alguns lugares são notificados menos de cinco novos casos por 100.000 habitantes por ano, enquanto há nações em que o valor de incidência ultrapassa 500 por 100.000 habitantes (WHO, 2019).

Em relação ao Brasil, ele figura entre os 30 países de alta carga para TB e TB-HIV, sendo considerado prioritário pela Organização Mundial da Saúde (OMS) para o controle da doença no mundo. $O$ país atingiu as metas relacionadas à incidência e mortalidade por TB, contribuindo para redução da carga da TB no mundo (WHO, 2015). Porém, em 2017, elevaram-se o número de casos diagnosticados e acompanhados no Sistema de Informação de Tratamentos 
Especiais de Tuberculose (SITE-TB) de multirresistência e polirresistência (BRASIL, 2019).

A transmissão da TB dá-se por inalação de gotículas de aerossol contendo o bacilo, com posterior deposição nos pulmões, levando a um dos quatro resultados possíveis: depuração imediata do organismo; doença primária, a qual é o início imediato da doença ativa; infecção latente ou doença de reativação, após um período de infecção latente (BRASIL, 2019).

O diagnóstico é clínico, bacteriológico e radiológico. O quadro clássico de sintomas apresenta-se com tosse persistente seca ou produtiva, febre vespertina, sudorese noturna e emagrecimento. A análise bacteriológica segue pelos exames de baciloscopia direta ou Teste Rápido Molecular para Tuberculose (TRM-TB). Já a investigação por imagem perpassa pela radiografia do tórax, método de escolha para avaliação inicial e acompanhamento, além da Tomografia Computadorizada (TC), recurso bastante sensível para demonstrar as alterações anatômicas, quando as informações na radiografia inicial são inconclusivas (BRASIL, 2019).

O tratamento envolve uma abordagem complexa que deve ser humanizada e com estabelecimento de vínculos, visto que a duração é extensa e requer aderência contínua do paciente, uma das maiores dificuldades observada na história da doença (BRASIL, 2019). Assim, decorre a importância da disseminação da informação acerca do assunto, pois a TB é uma doença curável em praticamente todos os casos sensíveis aos medicamentos anti-TB, desde que o tratamento seja realizado corretamente (WHO, 2019).

Nessa perspectiva, o uso de recursos audiovisuais pode aumentar o conhecimento populacional acerca do assunto. Isto posto, aulas, vídeos e reportagens jornalísticas de boa qualidade podem proporcionar maior educação e formação da população sobre o assunto e em decorrência, contribuir para a redução da transmissibilidade, seguimento correto e interino do tratamento e uma possível sobrevida do paciente.

Entre as possíveis ferramentas para a disseminação de informação em grande escala são as plataformas agregadoras de vídeos, como o YouTube. Sabese que atualmente muitas pessoas utilizam o sítio do Youtube para buscar 
conhecimento acerca de agravos à saúde. Nesse contexto, interroga-se: O que retratam os vídeos disponibilizados no YouTube sobre a Tuberculose?

Destarte, o objetivo desta pesquisa, considerando a relevância do assunto e, principalmente, a necessidade de constante atualização na área, foi analisar os vídeos no sítio de compartilhamento YouTube, observando cuidadosamente os pontos tratados nos vídeos relacionados à tuberculose com base no Manual de recomendações para o controle da tuberculose no Brasil (2019).

\section{METODOLOGIA}

A pesquisa caracteriza-se do tipo descritiva e abordagem quantitativa, realizada a partir da análise dos vídeos compartilhados no sítio Youtube, que possui o endereço virtual: www.youtube.com.

Para a realização do estudo, houve direcionamento metodológico através de um protocolo de pesquisa, o qual buscava refletir sobre como o tema da Tuberculose era abordado na plataforma digital.

A pesquisa foi realizada em fevereiro de 2020, por meio do descritor controlado "Tuberculose", extraído dos Descritores em Ciências da Saúde (DeCS). Foi utilizados os filtros "tipo de resultado: vídeo" e "Vídeo curto" oferecidos pelo próprio sítio, com ordenamento de acordo com o maior número de visualizações. Os links dos vídeos resultantes dessa busca inicial foram salvos para posterior análise, a fim de não comprometer a seleção da amostra, já que o sítio se caracteriza pela adição contínua de novos conteúdos.

Para a inclusão do vídeo nas pesquisas foram definidos os seguintes critérios: vídeos com referência direta a TB; vídeos em linguagem verbal - idioma português e vídeos postados no último ano. Foram excluídos os vídeos que não respondem à questão de pesquisa e/ou não se referem à temática ou estavam duplicados. Dessa forma, foram analisados os 300 primeiros vídeos, sendo a amostra final composta por 157 vídeos. 
Após a seleção dos vídeos, foi iniciada a coleta de dados. Para isto, foram assistidos os vídeos na íntegra para a extração das seguintes informações: tempo de duração; autor (pessoa física, órgão ou empresa); data da postagem; categoria (segundo classificação do YouTube); tipo do vídeo (educativo, jornalístico, outro); serviços institucionais envolvidos; profissionais envolvidos, temas relacionados à TB (patogênese, sinais e sintomas, tratamentos, prevenção) e presença de informação correta, de acordo com o Manual de recomendações para o controle da TB no Brasil.

Os dados obtidos foram tabulados, agrupados e analisados. Não se fez necessária a aprovação em comitê de ética, uma vez que a pesquisa não está envolvida diretamente com seres humanos, utilizando material de domínio público.

\section{RESULTADOS}

A sinopse dos dados referentes aos achados através da busca dos vídeos no sítio do youtube.com, está descrita na Figura 1. Estes, em sua maioria, são postados por empresas, majoritariamente do ramo jornalístico. Porém, encontram-se também vídeos postados por pessoa física, órgãos governamentais, bem como organizações não governamentais (ONGs) (Figura 1). 
Figura 1. Caracterização dos vídeos quanto a autoria, profissionais envolvidos, tempo de duração, tipo de vídeo e serviços institucionais envolvidos, Campina Grande, Paraíba, Brasil, 2020.

\begin{tabular}{|c|c|c|c|}
\hline \multicolumn{4}{|c|}{ Distribuição dos vídeos selecionados } \\
\hline Descritores & $\begin{array}{c}\text { Total de videos } N \text { - } \\
\text { (\%) }\end{array}$ & $\begin{array}{c}\text { Excluidos / } \\
\text { Repetidos N - (\%) }\end{array}$ & Selecionados $N$ - (\%) \\
\hline Tuberculous: & $181 * 100 \%$ & $27.1492 \%$ & $157-86.74 \%$ \\
\hline \multicolumn{4}{|c|}{ Autorin dos videos xelconados } \\
\hline Aatrolis. & & N & (8) \\
\hline tongres & & 86 & 8.78\% \\
\hline Orgta Govenamertil: & & 24 & $1529 \%$ \\
\hline oNC & & 3 & $191 \%$ \\
\hline 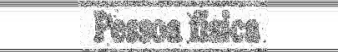 & & 4 & $28,08 \%$ \\
\hline 7n: & & 157 & Dowor: \\
\hline \multicolumn{4}{|c|}{ Principais profissionais envolvidos nos vídeos selecionados } \\
\hline Profissionais envolvidos & & $\mathbf{N}$ & $(\%)$ \\
\hline Enfermeiro & & 8 & $4,85 \%$ \\
\hline Jornalista & & 12 & $7,27 \%$ \\
\hline Médico & & 31 & $18,79 \%$ \\
\hline Dofesems & & 10: & $6.06 \%$ \\
\hline Gulvis? & & 10 & NWr \\
\hline Wul: & & 165 & Matre \\
\hline \multicolumn{4}{|c|}{ Tempo de duração dos vídeos selecionados } \\
\hline Tempo de duração & & N & $(\%)$ \\
\hline Menos de $2 \mathrm{~min}$ & & 62 & $39,49 \%$ \\
\hline 2 min oir mais & & $95^{\circ}$ & $60.51 \%$ \\
\hline lotil & & $15 \%$ & $100.01 \mathrm{j}^{\mathrm{i}}$ is \\
\hline \multicolumn{4}{|c|}{ Tipo do vídè selecionado } \\
\hline 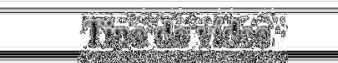 & & $x_{0}^{2}$ & 8 \\
\hline$=$ & & $=-1$ & $8 \sqrt{1}$ \\
\hline " & & S1: & (2) \\
\hline Outro & & 25 & $15,92 \%$ \\
\hline Total & & 157 & $100.00 \%$ \\
\hline
\end{tabular}

A figura 2 aborda os principais temas presentes nos vídeos inseridos. Depreende-se que um terço dos vídeos elaborados abordam o tratamento da TB, seguido pelos sinais e sintomas, prevenção da doença e visão geral da TB. 
Figura 2. Descrição dos vídeos quanto aos temas mais relevantes, Campina Grande, Paraíba, Brasil, 2020.

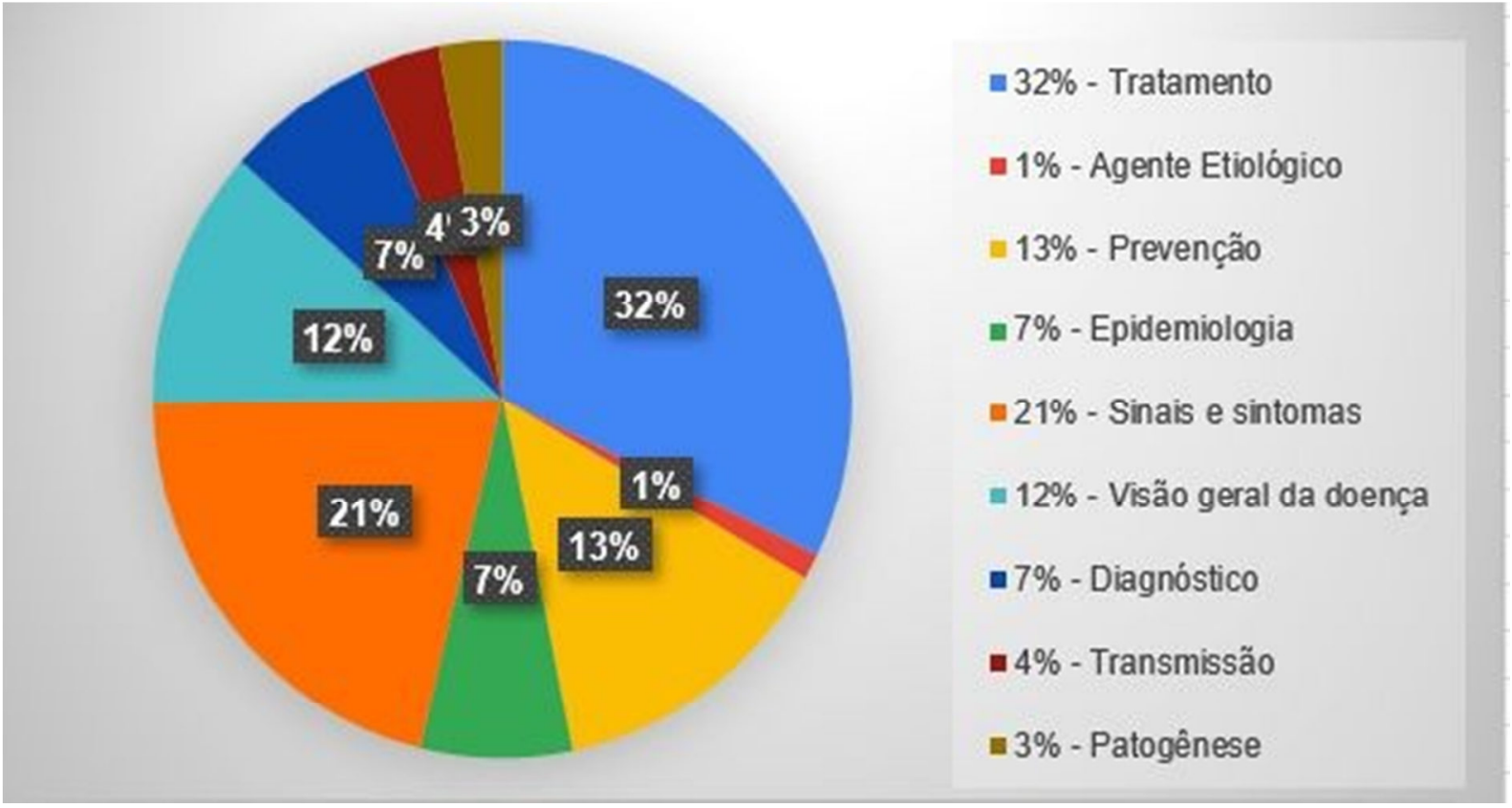

A figura 3 utiliza as informações presentes no Manual de recomendações para o controle da tuberculose no Brasil para averiguar a existência de informações corretas e relevantes. Percebe-se que $84 \%$ dos vídeos dispõem de informações importantes para a população.

Figura 3. Descrição dos vídeos quanto a presença de informações corretas e relevantes, Campina Grande, Paraíba, Brasil, 2020.

\begin{tabular}{|c|c|c|}
\hline \multicolumn{3}{|c|}{ Presença de informaçōes corretas e relevantes } \\
\hline 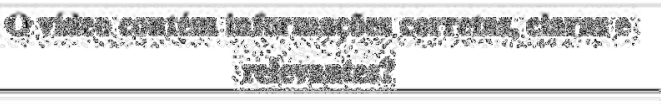 & 洫 & 16 \\
\hline Sim & 13\% & $84,08 \%$ \\
\hline Não & 21 & $13,38 \%$ \\
\hline Outro & 4 & $2,55 \%$ \\
\hline Total & 157 & $100,00 \%$ \\
\hline
\end{tabular}




\section{DISCUSSÃO}

A TB apesar de afetar a humanidade há 8.000 anos, ainda é muito mistificada. Embora seja uma patologia, que atualmente, é potencialmente curável e prevenível, ainda constitui como um grande problema de saúde (BRASIL, 2019). A desinformação é, por muitas vezes, o principal motivo para tantos casos de contágio que ainda ocorrem no país.

Dessa forma, procura-se sempre manter tentativas de criação de campanhas e formas de informatizar à população sobre a patologia, sendo um desses adventos utilizados a internet. A web vem contribuindo atualmente para a divulgação de conhecimento, uma vez que interliga uma grande quantidade de indivíduos em um curto espaço de tempo, é de fácil acesso e manuseio, e qualquer cidadão pode transportar informações, fatos, dados e notícias. Por meio dela o indivíduo tem acesso a muitas informações, as quais, permitem aprender e, consequentemente, agir, dialogar, debater, se mobilizar na defesa de direitos e participar de discussões públicas que afetem a sociedade (GRANZOTTO et al., 2017).

Dentre as principais plataformas digitais, está o YouTube, considerado o mais popular sítio de produtos audiovisuais, registrando mais de um bilhão de usuários em 2015, mostrando-se uma ferramenta de valor inquestionável para a sociedade contemporânea (BERNADAZZI et al., 2017).

Os vídeos existentes nesta plataforma abordam a TB de forma ampla, discutindo os aspectos mais importantes para a informação da população: sinais, sintomas, transmissão, diagnóstico, tratamento, prevenção, patogênese e dados epidemiológicos.

A maioria dos vídeos assistidos teve autoria de empresas, sobretudo jornais, sendo os órgãos governamentais responsáveis menos produtores de conteúdo. Assim, fica evidente, que o governo ainda precisa investir mais em vídeos sobre essa patologia, uma vez que a TBé uma das suas prioridades. O Ministério da Saúde, por meio da Coordenação-Geral do Programa Nacional de Controle da Tuberculose (CGPNCT), elaborou o plano nacional a fim de que a TB não seja um 
problema de saúde pública no Brasil (BRASIL, 2017). No entanto, para atingir esse objetivo, é necessário o planejamento de ações que busquem o acesso à prevenção, ao diagnóstico e ao tratamento. Nesse contexto, os vídeos educativos são meios eficazes na difusão do conhecimento de modo a auxiliar o governo nesse processo de redução da incidência da doença no país.

Os vídeos em sua maior parte apresentam caráter jornalístico e educativos. As notícias são textos expositivos informativos que pretendem transmitir as informações de modo objetivo, apenas alertando a população. E, embora seja apresentado muitos vídeos educativos, é importante que estes busquem ensinar ao público a importância da vacinação, sinais e sintomas da patologia, locais onde encontrar e realizar o tratamento adequado.

Além disso, a participação dos profissionais da saúde envolvidos com os vídeos foi pequena. Contudo, são estas pessoas que possuem um maior domínio do assunto, portanto deveriam ser mais participativos, ajudando a construir vídeos com informações mais coerentes, relevantes e corretas.

Dentre os tópicos abordados sobre o assunto global, o tratamento da TB foi o mais prevalente. É um assunto que ainda precisa ser muito discutido, pois embora a eficácia do esquema antituberculose seja de até 95\%, a efetividade do tratamento depende da adesão dos pacientes (RABAHI et al., 2017). Estes, muitas vezes, não conhecem de forma adequada o tratamento e o abandonam por apresentarem uma melhora clínica, e ainda há aqueles que fazem o uso errado dos medicamentos ou o uso irregular (BRASIL, 2019).

Outra temática abordada foi a prevenção. Sabe-se que os recursos audiovisuais trazem informações sobre a vacina BCG, quimioprofilaxia e medidas de higiene, mas, de modo associado, deveria ser mais produzido conteúdo sobre sinais e sintomas, pois estes que permitem reconhecer rapidamente o indivíduo com TB para tratá-lo e prevenir a transmissão.

Como a maioria dos vídeos entraram na categoria jornalística, a temática epidemiológica demonstra-se presente como um dos tópicos mais relacionados à TB. Esses dados mostraram-se essenciais para reconhecer a quão expandida está a doença no Brasil e permitem perceber sua incidência com mais intensidade em 
locais específicos, como por exemplo, na população privada de liberdade, no sistema prisional.

Um estudo realizado no Rio de Janeiro revelou que $84 \%$ dos doentes adquiriram a tuberculose na prisão (BRASIL, 2016). Isso sugere que a TB entre as pessoas privadas de liberdade está predominantemente relacionada à transmissão intrainstitucional massiva ligada às precárias condições de encarceramento na maioria das prisões brasileiras (BRASIL, 2019). Essas informações ajudam a divulgar e esclarecer sobre a doença, identificar situações críticas e promover ações de apoio aos que estão mais vulneráveis (BOGA, 2014).

Dentre os outros temas relacionados à TB, é válido ressaltar que há poucos vídeos sobre a transmissão da doença (Figura 2), algo que deveria ser reconsiderado, pois ainda existe muito desconhecimento populacional de como ela ocorre. De acordo Ferreira (2014), devido à falta de conhecimento, muitas pessoas acabam mudando seus hábitos, isolando pratos, talheres copos e toalhas, o que contribui para que a doença ainda seja estigmatizada. Um dos vídeos assistidos, "Tuberculose, combatendo o estigma" (MSF BRASIL, 2019), retrata o preconceito sofrido pelos pacientes e a necessidade do cuidado humanizado para que eles se sintam apoiados e continuem o tratamento.

Nesse âmbito, é necessário que os cidadãos entendam como se dá a transmissividade para realizar práticas simples, tais quais: deixar os ambientes abertos e ensolarados, pois o bacilo é sensível à luz solar, e a circulação de ar possibilita a dispersão de partículas infectantes (BRASIL, 2019).

Por fim, com os dados retratados na Figura 3, percebeu-se que os vídeos, em sua grande maioria, apresentaram informações corretas, claras e relevantes, o que gera um impacto positivo para o conhecimento daqueles que os assistem. Isso reforça a perspectiva de que o YouTube é uma plataforma digital que dispõe de conteúdos importantes e pode transferir confiabilidade para os internautas. No entanto, apesar de pequena, há ainda uma parcela de vídeos que mostrou conteúdo sem comprovação científica, hesitantes e até mesmo incorretos.

Dessa forma, embora a plataforma de vídeos contribua no processo de aprendizagem, não há um controle rigoroso quanto ao conteúdo veiculado em seus 
vídeos, sendo necessário, portanto, que os cidadãos usem essa ferramenta com sabedoria a fim de aproveitar positivamente as temáticas disponibilizadas.

\section{CONCLUSÃO}

O Youtube é uma das mais importantes plataformas digitais para disseminação de conteúdo audiovisual na atualidade, sendo então uma ferramenta muito útil quando utilizada para propagação de informações de saúde para a sociedade contemporânea. Esse sítio de conhecimento possui um acervo amplo de vídeos relacionados a doença TB, em sua grande maioria de caráter jornalístico, de autoria de empresas, sobretudo de Jornais, apresentando o tratamento e a prevenção da TB como os temas mais prevalentes.

Ainda nesse contexto, é notória a objetividade das informações, a fim de promover um alerta para população em um curto espaço de tempo. Apesar de majoritariamente apresentarem dados corretos, claros e relevantes, observa-se que nos vídeos disponíveis o contexto educacional é muitas vezes negligenciado, além de haver pouca participação de profissionais da área da saúde, os quais possuem maior domínio do assunto. Ademais, ainda há pouco conteúdo relacionado aos sinais e sintomas, diagnóstico e patogênese da doença.

Tendo em vista os resultados obtidos, pôde-se concluir que o governo precisa investir mais em vídeos educativos sobre essa patologia, levando-se em consideração que a TB é uma de suas prioridades e que esse tipo de conteúdo é eficaz na disseminação de conhecimento, de forma a reduzir a incidência da doença no país. Por fim, apesar de comporem uma pequena parcela, há vídeos que mostram dados sem comprovação científica, hesitantes e até mesmo incorretos. Portanto, é imprescindível o uso racional dessa ferramenta a fim de que haja o aproveitamento positivo de todas as temáticas disponibilizadas. 


\section{REFERÊNCIAS BIBLIOGRÁFICAS}

BERNADAZZI, R.; COSTA, M. H. B. e V. Produtores de conteúdo no YouTube e as relações com a produção audiovisual. Revista Communicare. Disponível em: https://casperlibero.edu.br/wp-content/uploads/2017/09/Artigo-7-Communicare-17-Edi\%C3\% A7\%C3\%A3o-Especial.pdf . Acesso em: 28 fev. 2020.

BRASIL. Ministério da Saúde. Manual de Recomendações para o Controle da Tuberculose no Brasil. Secretaria de Vigilância em Saúde. Programa Nacional de Controle da Tuberculose. Brasília: Ministério da Saúde; 2019. Disponível em: http://bvsms.saude.gov.br/bvs/publicacoes/manual_recomendacoes_controle_tuberculose_bra sil_2_ed.pdf. Acesso em: 25 fev. 2020.

BRASIL. Ministério da Saúde. Secretaria de Vigilância em Saúde. Departamento de Vigilância das Doenças Transmissíveis. Brasil Livre da Tuberculose: Plano Nacional pelo Fim da Tuberculose como Problema de Saúde Pública. Ministério da Saúde, Secretaria de Vigilância em Saúde, Departamento de Vigilância das Doenças Transmissíveis. Brasília: Ministério da Saúde, $2017 . \quad$ Disponível em: http://bvsms.saude.gov.br/bvs/publicacoes/brasil_livre_tuberculose_plano_nacional.pdf. Acesso em: 25 fev. 2020.

CARVALHO, F. J. C. et al. Economia monetária e financeira: teoria e política. 2. ed. rev. e atual. Rio de Janeiro: Elsevier: Campus, 2007.

FERREIRA, Kuitéria Ribeiro. A adesão ao tratamento no caso da tuberculose multirresistente. 2014. $281 \mathrm{f}$. Tese (Doutorado) - Curso de Enfermagem, Universidade de São Paulo, São Paulo, 2014. Disponível em: https://www.teses.usp.br/teses/disponiveis/7/7141/tde16042015-163038/publico/kuiteria_ribe iro_ferreira_tese_final_versao_corrigida.pdf. Acesso em: 29 fev. 2020.

GRANZOTTO, I.T. et al. A internet na sociedade da informação: promovendo a sustentabilidade e protegendo o meio ambiente. Santa Maria, nov. 2017. Disponível em: < http://coral.ufsm.br/congressodireito/anais/2017/4-6.pdf >. Acesso em: 28 fev. 2020.

RABAHI, Marcelo Fouad et al. Tuberculosis treatment. Jornal Brasileiro de Pneumologia. São Paulo, p. 472-486. dez. 2017. Disponível em: http://www.scielo.br/pdf/jbpneu/v43n6/pt_18063713-jbpneu-43-06-00472.pdf. Acesso em: 29 fev. 2020.

SAAVACOOL, J. Philadelphia and the white plague. Philadelphia: Trans Stud Coll Physicians, 1986 , v. 8 , p. $147-182$.

SALVADOR, Pétala Tuani Candido de Oliveira et al . Segurança do paciente: caracterização de vídeos do YouTube. Rev. Gaúcha Enferm., Porto Alegre , v. 38, n. 1, e61713, 2017. Disponivel em: http://www.scielo.br/scielo.php?script=sci_arttext\&pid=S198314472017000100402\&lng=en \&nrm=iso. Acesso em: 28 fev. 2020.

TUBERCULOSE COMBATENDO O ESTIGMA. [S. I.: s. n.], 2019. 1 vídeo (2 min). Publicado pelo canal MSF BRASIL. Disponível em: https://www.youtube.com/watch?v=mnZKVYgq8I4. Acesso em: 29 mar. 20.

WHO, WORLD HEALTH ORGANIZATION. Global Tuberculosis Report. 2019. Disponível em: https://apps.who.int/iris/bitstream/handle/10665/329368/9789241565714-eng.pdf?ua=1. Acesso em: 27 fev. 2020. 